\section{(6) \\ OPEN ACCESS}

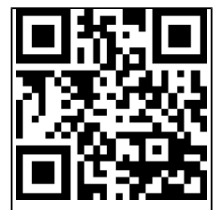

Open Access Scan to access more free content

- Additional material is published online only. To view please visit the journal online (http://dx.doi.org/10.1136/ thoraxjnl-2015-207728).

${ }^{1}$ Division of Allergy, Pulmonary, and Critical Care Medicine, Vanderbilt University School of Medicine, Nashville, Tennessee, USA

${ }^{2}$ Department of Pathology, Microbiology, and Immunology, Vanderbilt University School of Medicine, Nashville, Tennessee, USA

${ }^{3}$ Department of Neurology, Vanderbilt University, Nashville, Tennessee, USA

\section{Correspondence to}

Dr R Stokes Peebles, Vanderbilt University Medical Center,

1161 21st Avenue, T-1218 MCN, Nashville TN 372322650, USA; stokes.peebles@ vanderbilt.edu

An abstract of this work was presented at the American Academy of Allergy, Asthma, and Immunology Annual Meeting in Los Angeles, CA on 6 March 2016

Received 19 August 2015 Revised 25 February 2016 Accepted 1 March 2016 Published Online First 12 April 2016

\title{
The histone deacetylase inhibitor trichostatin A suppresses murine innate allergic inflammation by blocking group 2 innate lymphoid cell (ILC2) activation
}

\author{
Shinji Toki, ${ }^{1}$ Kasia Goleniewska, ${ }^{1}$ Sara Reiss, ${ }^{1}$ Weisong Zhou, ${ }^{1}$ Dawn C Newcomb, ${ }^{1,2}$ \\ Melissa H Bloodworth, ${ }^{2}$ Matthew T Stier, ${ }^{2}$ Kelli L Boyd, ${ }^{2}$ Vasiliy V Polosukhin, ${ }^{1}$ \\ Sriram Subramaniam, ${ }^{3} \mathrm{R}$ Stokes Peebles $\mathrm{Jr}^{1,2}$
}

\section{ABSTRACT}

Background Group 2 innate lymphoid cells (ILC2) are an important source of the type 2 cytokines interleukin (IL)-5 and IL-13 that are critical to the allergic airway phenotype. Previous studies reported that histone deacetylase (HDAC) inhibition by trichostatin A (TSA) downregulated adaptive allergic immune responses; however, the effect of HDAC inhibition on the early innate allergic immune response is unknown. Therefore, we investigated the effect of TSA on innate airway inflammation mediated by ILC2 activation.

Methods $\mathrm{BALB} / \mathrm{c}$ mice were challenged intranasally with Alternaria extract, exogenous recombinant mouse IL-33 (rmIL-33) or the respective vehicles for four consecutive days following TSA or vehicle treatment. Bronchoalveolar lavage (BAL) fluids and lungs were harvested $24 \mathrm{~h}$ after the last challenge.

Results We found that TSA treatment significantly decreased the number of ILC2 expressing IL-5 and IL-13 in the lungs challenged with Alternaria extract or rmIL33 compared with vehicle treatment $(p<0.05)$. TSA treatment significantly decreased protein expression of IL-5, IL-13, CCL11 and CCL24 in the lung homogenates from Alternaria extract-challenged mice or rmIL-33challenged mice compared with vehicle treatment $(p<0.05)$. Further, TSA treatment significantly decreased the number of perivascular eosinophils and mucus production in the large airways that are critical components of the asthma phenotype $(p<0.05)$. TSA did not change early IL-33 release in the BAL fluids; however, TSA decreased lung IL-33 expression from epithelial cells $24 \mathrm{~h}$ after last Alternaria extract challenge compared with vehicle treatment $(p<0.05)$.

Conclusions These results reveal that TSA reduces allergen-induced ILC2 activation and the early innate immune responses to an inhaled protease-containing aeroallergen.

\section{INTRODUCTION}

CrossMark

\section{To cite: Toki $S$,}

Goleniewska K, Reiss S,

et al. Thorax 2016;71:

633-645.
Asthma is a chronic lung disorder that is characterised by inflammation and swelling in the airway that results in mild-to-severe breathing difficulty during an exacerbation. ${ }^{1}$ The mechanisms that initiate allergic immune responses in asthma development are incompletely understood. The T-helper (Th) 2 cytokines, IL-4, IL-5, IL-9 and IL-13 are
Key messages

What is the key question?

- Does histone deacetylase (HDAC) inhibition suppress the early immune response to an inhaled aeroallergen by blocking type 2 immune responses mediated by group 2 innate lymphoid cells (ILC2)?

What is the bottom line?

- Trichostatin A downregulated the number of ILC2 expressing IL-5 and IL-13 following Alternaria extract challenge with consequent attenuation of lung eosinophillia and mucus hypersecretion.

\section{Why read on?}

- This is the first report that an HDAC inhibitor suppressed innate immune-mediated allergic inflammation, identifying a novel target for therapeutics in allergen-induced lung diseases.

critical in the pathogenesis of allergic asthma as these cytokines regulate the Th2-type response including B cell class switching to IgE production, eosinophilia, mucus production, and bronchoconstriction. $^{2}{ }^{3}$ The main source of these cytokines was long considered to be Th2 cells produced in the setting of adaptive immune responses. Recently, alternative ways to induce Th2 cytokine production have been discovered in both mouse and human studies. $^{4-6}$ Group 2 innate lymphoid cells (ILC2) are resident in the lung and are also an important source of IL-5 and IL-13. ILC2 are lineage negative $\left(\mathrm{Lin}^{-}\right)$cells that do not have cell surface expression of T cell, B cell, macrophage, dendritic cell (DC), neutrophil or natural killer (NK) cell markers, yet these cells have surface expression of ST2 (IL-33 receptor), CD25 (IL-2R $\alpha$ ) and CD127 (IL-7R $\alpha){ }^{5}$ ILC2 are activated by epithelial-derived cytokines such as IL-33, TSLP, IL-25 or their combination, which results in ILC2 production of IL-5 and IL-13. In vivo mouse studies revealed that proteases or aeroallergens containing protease activity, such as Alternaria alternata induced IL-33 release from 
type II epithelial cells. ${ }^{7}$ In addition, exogenous IL-33 administration induced Th2-type airway inflammation mediated by ILC2, but not CD4 ${ }^{+}$T cells. $^{8}$

Epigenetic regulation of gene expression has been reported as a novel approach to treat allergy and other immune-mediated diseases. ${ }^{9} 10$ One epigenetic mechanism for regulating gene expression is the acetylation and deacetylation of histone by histone acetyltransferases (HATs) and histone deacetylases (HDACs), respectively. The acetylation of histones by HATs increases DNA accessibility and promotes gene expression, whereas the removal of acetyl groups by HDACs has the opposite effect. ${ }^{11}$ There are 11 isoforms of HDACs and there are also many non-histone protein substrates of HDACs in addition to histone proteins. ${ }^{12}$ Compounds that have the ability to inhibit multiple HDAC isoforms are called pan-HDAC inhibitors (HDACi). Currently, there are three pan-HDACi drugs, vorinostat, romidepsin, and belinostat, that have been approved by the US Food and Drug Administration (FDA) for cancer treatment. ${ }^{13}$ Trichostatin A (TSA) is not approved by FDA, but is a pan-HDACi that inhibits enzyme activity of the HDAC 1, 2, 3, 4, 6 and 10 isoforms.

Several studies have indicated that TSA enhances inflammatory gene expression mediated by nuclear factor-kappa B $(\mathrm{NF}-\kappa \mathrm{B}) .{ }^{14-16}$ In contrast, many studies indicate that HDACi suppressed expression of proinflammatory genes. ${ }^{9}$ For instance, TSA repressed lipopolysaccharide (LPS)-induced protein expression of TNF- $\alpha$, IL- 6 , IL- $1 \beta^{17}$ and IL- $12 \mathrm{p} 40^{18}$ in bone marrow derived macrophages. Further, TSA suppressed LPS-induced or poly I:C-induced mRNA expression of proinflammatory cytokines and chemokines in human microglial cells. ${ }^{19}$ In in vivo experiments, TSA administration during antigen sensitisation or challenge suppressed ovalbumin (OVA)-induced airway inflammation and hyperresponsiveness in a mouse model dependent upon adaptive immune responses ${ }^{20} 21$ and ameliorated airway constriction in an Aspergillus fumigatus antigen-sensitised and challenged allergic mouse model. ${ }^{22}$ However, no studies have investigated the effect of HDACi on the innate allergic inflammatory response that is mediated by ILC2.

We hypothesised that HDAC inhibition negatively regulates innate allergic immune responses through suppression of ILC2 function. To test this hypothesis, we investigated the effects of HDAC inhibition by TSA on Alternaria extract-induced innate allergic inflammation model in mice. In this study, we found that TSA treatment significantly decreased the number of ILC2-expressing IL-5 and IL-13 in the lungs of mice challenged with Alternaria extract compared with vehicle treatment. In addition, TSA treatment decreased protein expression of IL-5, IL-13, CCL11 (eotaxin) and CCL24 (eotaxin-2) in the bronchoalveolar lavage (BAL) fluid and lung homogenates compared with vehicle treatment. Moreover, TSA treatment significantly decreased the number of perivascular eosinophils and mucus production in the large airways that are critical components of the allergic asthma phenotype. Furthermore, TSA treatment significantly decreased the number of ILC2-expressing IL-5 and IL-13 in the lungs and the protein expression of IL-5, IL-13, CCL11 and CCL24 in the lung homogenates of mice challenged with exogenous recombinant mouse IL-33 (rmIL-33) compared with vehicle treatment.

\section{METHODS}

\section{Mice}

Eight-week to 10-week-old female wild-type (WT) BALB/c mice were obtained from Charles River Laboratories. IL-33 Citrine/+ reporter mice were generated by crossbreeding WT BALB/c mice and IL-33 ${ }^{\text {Citrine/Citrine }}$ mice. ${ }^{23}$ Animal experiments were approved by the Institutional Animal Care and Use Committee at Vanderbilt University and were conducted according to the guidelines for the Care and Use of Laboratory Animals prepared by the Institute of Laboratory Animal Resources, National Research Council.

\section{HDAC inhibitor treatment and Alternaria extract challenge or recombinant mouse IL-33 challenge}

TSA $(2.0 \mathrm{mg} / \mathrm{kg}$ in $50 \mu \mathrm{L}$ dimethyl sulfoxide (DMSO); Selleckchem) or $50 \mu \mathrm{L}$ DMSO as vehicle was administered subcutaneously to mice anaesthetised with isoflurane daily for 2 days prior to either Alternaria extract challenge (GREER), rmIL-33 challenge (Peprotech) or the respective vehicle challenge (figures 1A, 4A and 6A) and continued until the day prior to harvest. From day 0 to day 3 , either $5 \mu \mathrm{g}$ (protein amount) of Alternaria extract in $100 \mu \mathrm{L}$ of phosphate buffered saline (PBS), $0.5 \mu \mathrm{g}$ of rmIL-33 in $100 \mu \mathrm{L}$ of $0.1 \%$ BSA/PBS or $100 \mu \mathrm{L}$ of respective vehicles was used as an intranasal challenge to mice anaesthetised with ketamine/xylazine $1 \mathrm{~h}$ after TSA or its vehicle treatment. In an additional experiment, TSA or its vehicle was administered concomitantly with Alternaria extract challenge or PBS challenge (figure 8A). One hour or $24 \mathrm{~h}$ after the last challenge of Alternaria extract, rmIL-33 or the respective vehicles, the mice were sacrificed and BAL fluid and whole lungs were harvested for cytokine and chemokine measurements.

\section{Flow cytometry}

Lungs were harvested $24 \mathrm{~h}$ after the last challenge of Alternaria extract, rmIL-33 or the respective vehicles. The lungs were minced and digested with collagenase IV $(1 \mathrm{mg} / \mathrm{mL}$, SigmaAldrich) and DNase I (50 IU/mL, Sigma-Aldrich) in RPMI1640 with $5 \%$ fetal bovine serum (FBS) for $1 \mathrm{~h}$ at $37^{\circ} \mathrm{C}$, and a single cell suspension was obtained by grinding and passing the digested lung through a $70 \mu \mathrm{m}$ cell strainer. The cells were stimulated with phorbal 12-myristate 13-acetate (PMA) (10 ng/ $\mathrm{mL})$, ionomycin $(1 \mu \mathrm{M})$ and BD GolgiStop (BD Biosciences) at the manufacturer's recommended concentration for $6 \mathrm{~h}$. Then, both cell surface and intracellular cytokine staining were performed to enumerate $\mathrm{IL}-5^{+}$and $\mathrm{IL}-13^{+}$ILC2 and CD4T cells. ILC2 were identified as $\mathrm{Lin}^{-} \mathrm{CD} 45^{+} \mathrm{CD} 25^{+} \mathrm{CD} 127^{+}$cells. $\mathrm{CD}^{+}{ }^{+} \mathrm{T}$ cells were identified as $\mathrm{CD}^{+}{ }^{+} \mathrm{CD} 4^{+}$cells. To determine the IL-33-expressing cells (citrine reporter positive), cell surface staining was performed to distinguish the type of white blood cells and epithelial cells. $\mathrm{CD}^{+} \mathrm{T}$ cells were identified as $\mathrm{CD}^{+} \mathrm{CD}^{+}$cells. B cells were identified as $\mathrm{CD} 3^{-} \mathrm{CD} 19^{+}$ cells. Macrophages were identified as $\mathrm{F} 4 / 80^{+} \mathrm{CD} 11 \mathrm{c}^{\text {high }}$ cells. DCs were identified as F4/80- $\mathrm{CD}^{-} 1 \mathrm{c}^{+}$cells. Eosinophils were identified as siglec $\mathrm{F}^{+} \mathrm{Gr}-1^{\text {int }}$ cells. Granulocytes were identified as Gr- $1^{\text {high }}$ cells. Epithelial cells were identified as CD45EpCAM $^{+}$cells. All samples were measured on a BD LSR II Flow Cytometer and analysed using FlowJo Software.

\section{Cytokine measurements}

Quantikine ELISA or DuoSet ELISA kits from R\&D were used to measure the concentration of cytokines and chemokines in BAL fluids and lung homogenates according to manufacturer's instructions. Values below the detection limit were assigned a value at half of the lower limit of detection to allow for statistical analysis.

\section{Cell differential counting}

Cytospin preparations of BAL fluid cells were performed for each sample. The slides were stained with a Richard-Allan 
A TSA or vehicle s.c.

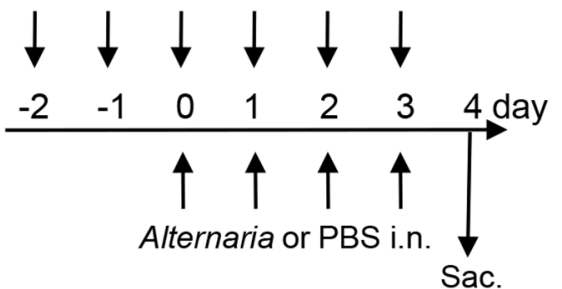

B

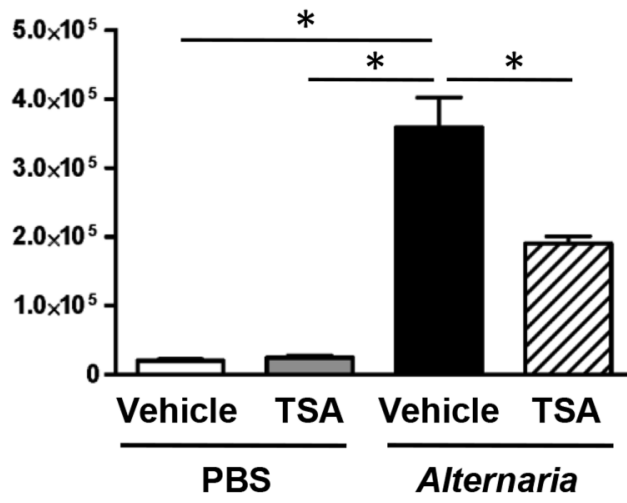

Number of IL-13+ ILC2

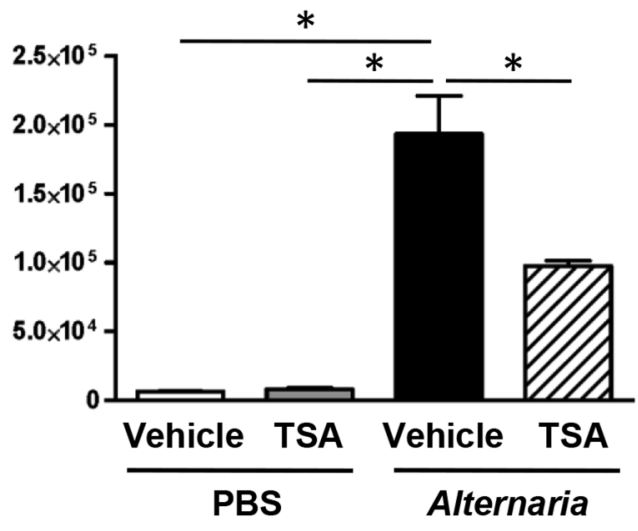

C Vehicle-PBS TSA-PBS Vehicle-Alternaria TSA-Alternaria
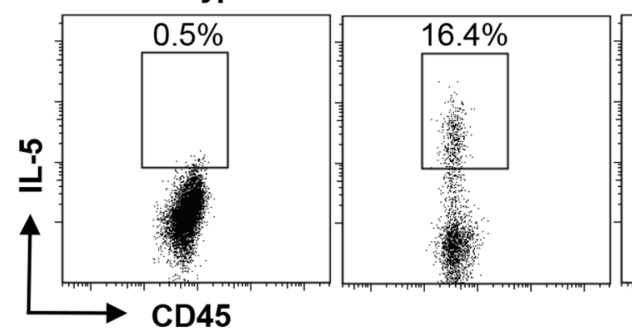

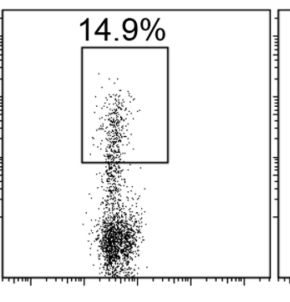
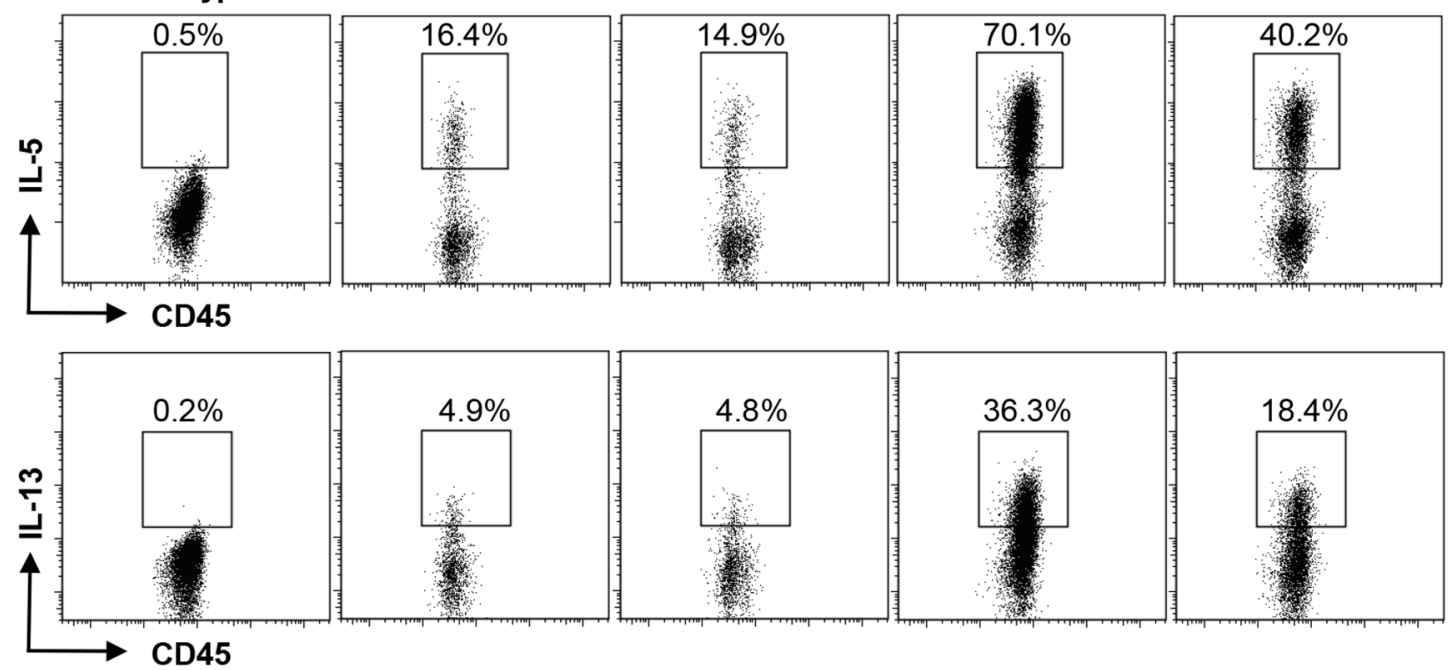

Figure 1 (A) Mice were challenged with Alternaria extract intranasally for four consecutive days. Trichostatin A (TSA) or its vehicle was administered subcutaneously on day -2 and -1 , and then every $1 \mathrm{~h}$ before Alternaria extract challenge on day $0-3$. (B) The number of IL-5-expressing and IL-13-expressing group 2 innate lymphoid cell (ILC2) in the lung from the mice challenged with PBS or Alternaria extract following vehicle or TSA treatment. (C) Representative plots of IL-5 and IL-13 intracellular staining in ILC2 in the lungs of mice challenged with PBS or Alternaria extract following vehicle or TSA treatment. The results are shown as mean \pm SEM of four mice in PBS-challenged groups and six mice in Alternaria extract-challenged groups. ${ }^{*} \mathrm{p}<0.05$.

Scientific Three-Step Stain Kit (Thermo Scientific) to determine cell differentials. Differential counts were based on counting 200 cells per slide.

\section{Histological analyses of lung sections}

Twenty-four hours after the last challenge of Alternaria extract or PBS, mice were sacrificed and the lung tissue was fixed in $10 \%$ formalin solution, paraffin-embedded, cut in $6 \mu \mathrm{m} \mathrm{sec-}$ tions, mounted and stained with anti-major basic protein (MBP) antibody generously provided by Dr. James J. Lee (Mayo Clinic, Scottsdale, Arizona, USA) to evaluate eosiniophils. ${ }^{24}$ Slides were examined by a pathologist blinded to experimental groups to score eosinophil infiltration using a $0-4$ scoring system: 0 , no eosinophils; 1, a few circulating eosinophils and rarely one or two eosinophils are seen in perivascular spaces; 2 , increased circulating eosinophils and eosinophils are prominent in perivascular spaces of large to medium vessels; 3 , circulating eosinophils are prominent and eosinophilic perivascular cuffs are observed 

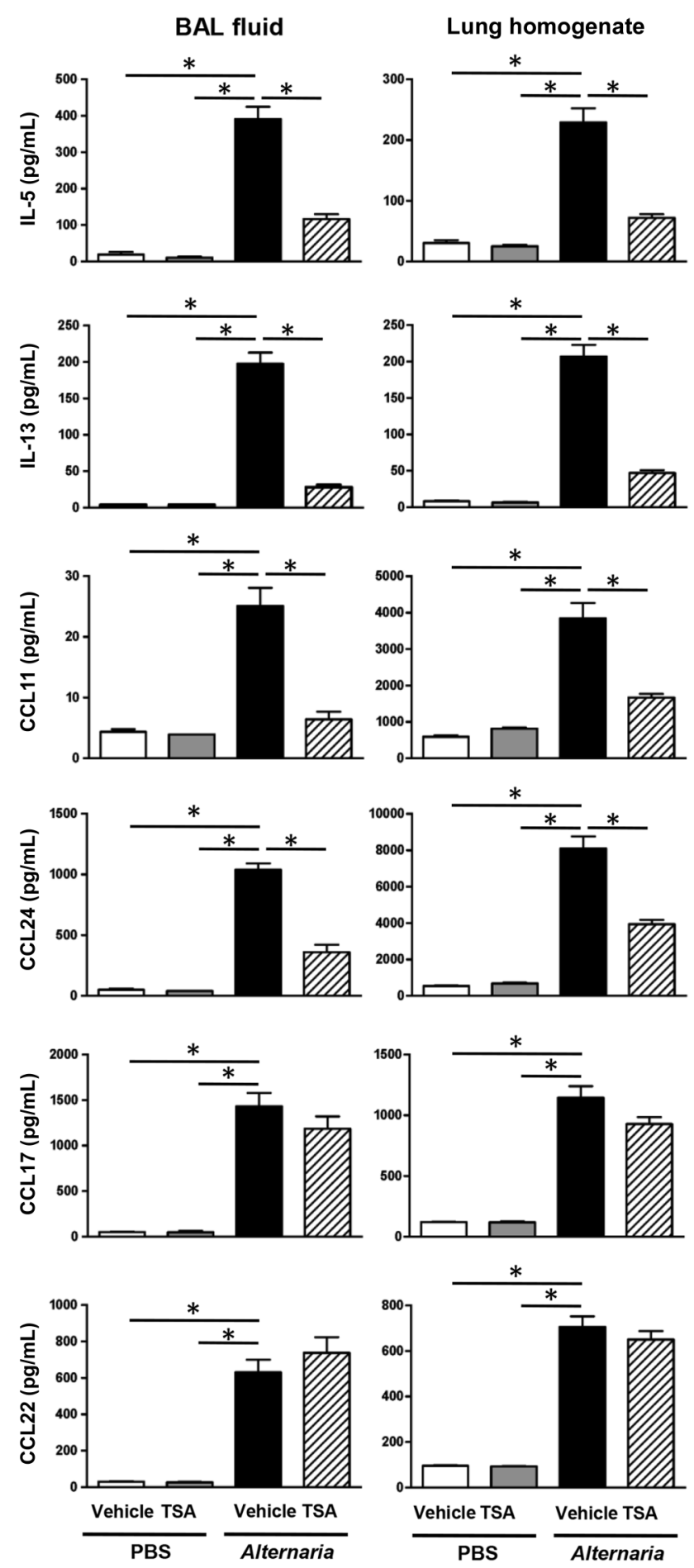

Figure 2 Bronchoalveolar lavage (BAL) fluids and lungs were harvested $24 \mathrm{~h}$ after the last Alternaria extract challenge to measure the protein expression of IL-5, IL-13, CCL11, CCL17, CCL22 and CCL24. The results are shown as mean \pm SEM of four mice in PBS-challenged groups and six mice in Alternaria extract-challenged groups. ${ }^{*} \mathrm{p}<0.05$.

in large, medium and small vessels; 4, increased circulating eosinophils, prominent perivascular cuffing by eosinophils in all vessels and interstitial infiltrates of eosinophils.

Paraffin-embedded lung tissue slices from mice sacrificed $48 \mathrm{~h}$ after the last challenge of Alternaria extract or PBS were stained with periodic acid-Schiff's (PAS) solution to visualise mucus and mucous-producing cells. Slides were scored by a pathologist blinded to the experimental groups. All airways in the section were scored (15-40 airways) according to a five-point scale: 0 , no PAS-positive cells; 1, <5\% PAS-positive cells; 2, 5-10\% PAS-positive cells; 3, 10-25\% PAS-positive cells and 4, >25\% PAS-positive cells.

\section{Statistical analysis}

All data were reported as mean \pm SEM. The data were compared by one-way analysis of variance (ANOVA) with Bonferronimultiple pairs comparisons test using GraphPad Prism 5 (GraphPad Software). $\mathrm{p}$ Values $<0.05(\mathrm{p}<0.05)$ were considered significant between two groups.

\section{RESULTS}

TSA treatment suppresses the number of mouse lung ILC2-producing IL-5 and IL-13 in response to airway Alternaria extract challenge

To establish the effect of TSA on cytokine production by ILC2, we enumerated the number of IL-5-expressing and IL-13-expressing lung ILC2 following either four consecutive days of PBS or Alternaria extract challenge in TSA-treated or vehicle-treated mice. The gating strategy for ILC2 is shown in online supplementary figure S1. ILC2 was identified as $\mathrm{Lin}^{-}$ $\mathrm{CD} 45^{+} \mathrm{CD} 25^{+} \mathrm{CD} 127^{+}$cell. Alternaria extract challenge significantly increased the number of lung ILC2 that expressed either IL-5 $\quad(\mathrm{p}<0.05)$ or IL-13 $(\mathrm{p}<0.05)$ compared with PBS-challenged mice (figure $1 \mathrm{~B}$ ). There was a statistically significant decrease in the number of IL-5-expressing and IL-13-expressing ILC2 $(\mathrm{p}<0.05)$ in the Alternaria extract-challenged mice treated with TSA compared with the Alternaria extract-challenged mice treated with vehicle for TSA (figure 1B). Figure 1C shows the representative flow cytometry dot plots of IL-5 and IL-13 intracellular staining in the lung ILC2 from mice challenged with PBS or Alternaria extract following TSA or vehicle treatment.

To determine the contribution of $\mathrm{CD}^{+}{ }^{+} \mathrm{T}$ cells to the IL-5 and IL-13 expression $24 \mathrm{~h}$ after four consecutive days of Alternaria extract challenge, intracellular cytokine staining was performed by flow cytometry (see online supplementary figure S2). The number of $\mathrm{CD} 3{ }^{+} \mathrm{CD} 4{ }^{+} \mathrm{IL}-5^{+}$and $\mathrm{CD} 3{ }^{+} \mathrm{CD} 4{ }^{+} \mathrm{IL}-13^{+}$cells was significantly increased in Alternaria extract-challenged groups compared with PBS-challenged groups $(\mathrm{p}<0.05)$, while no change was detected in the number of $\mathrm{CD} 3{ }^{+} \mathrm{CD} 4{ }^{+} \mathrm{IL}-5^{+}$ and $\mathrm{CD} 3{ }^{+} \mathrm{CD} 4{ }^{+} \mathrm{IL}-13^{+}$cells between TSA-treated group and vehicle-treated group in Alternaria extract-challenged mice. Online supplementary figure S2B shows the representative flow cytometry dot plots of IL-5 and IL-13 intracellular staining in the lung $\mathrm{CD}^{+}{ }^{+} \mathrm{CD} 4{ }^{+}$T cells from mice challenged with PBS or Alternaria extract following TSA or vehicle treatment. In the Alternaria extract-challenged groups, there was a statistically significant 12-fold increase in the number of IL-5-expressing ILC2 $(\mathrm{p}<0.05) \quad$ (figure 1B) compared with IL-5-expressing $\mathrm{CD} 3{ }^{+} \mathrm{CD} 4{ }^{+}$cells (see online supplementary figure S2A), and a 2.5 -fold increase in the number of IL-13-expressing ILC2 $(\mathrm{p}<0.05) \quad$ (figure 1B) compared with IL-13-expressing $\mathrm{CD} 3{ }^{+} \mathrm{CD}^{+}{ }^{+}$cells (see online supplementary figure S2A). These results indicate that ILC2, but not $\mathrm{CD}^{+}{ }^{+} \mathrm{CD} 4{ }^{+} \mathrm{T}$ cells are the major source of IL-5 and IL-13 in response to this four consecutive days airway Alternaria extract-challenge protocol.

TSA treatment suppresses Alternaria extract-induced cytokine and chemokine expression in the lung and airway We found that Alternaria extract challenge increased the number of IL-5-expressing and IL-13-expressing ILC2, and TSA 
A

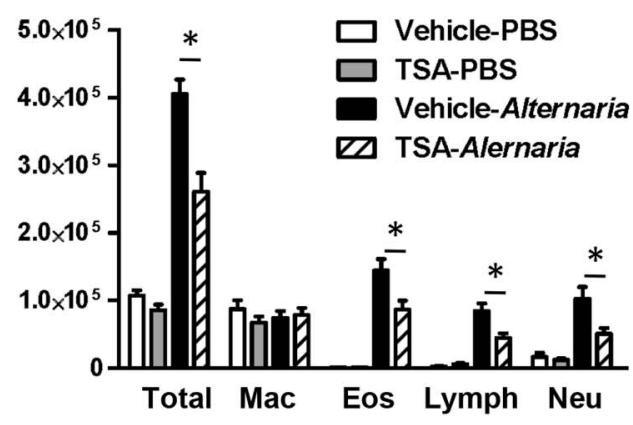

B
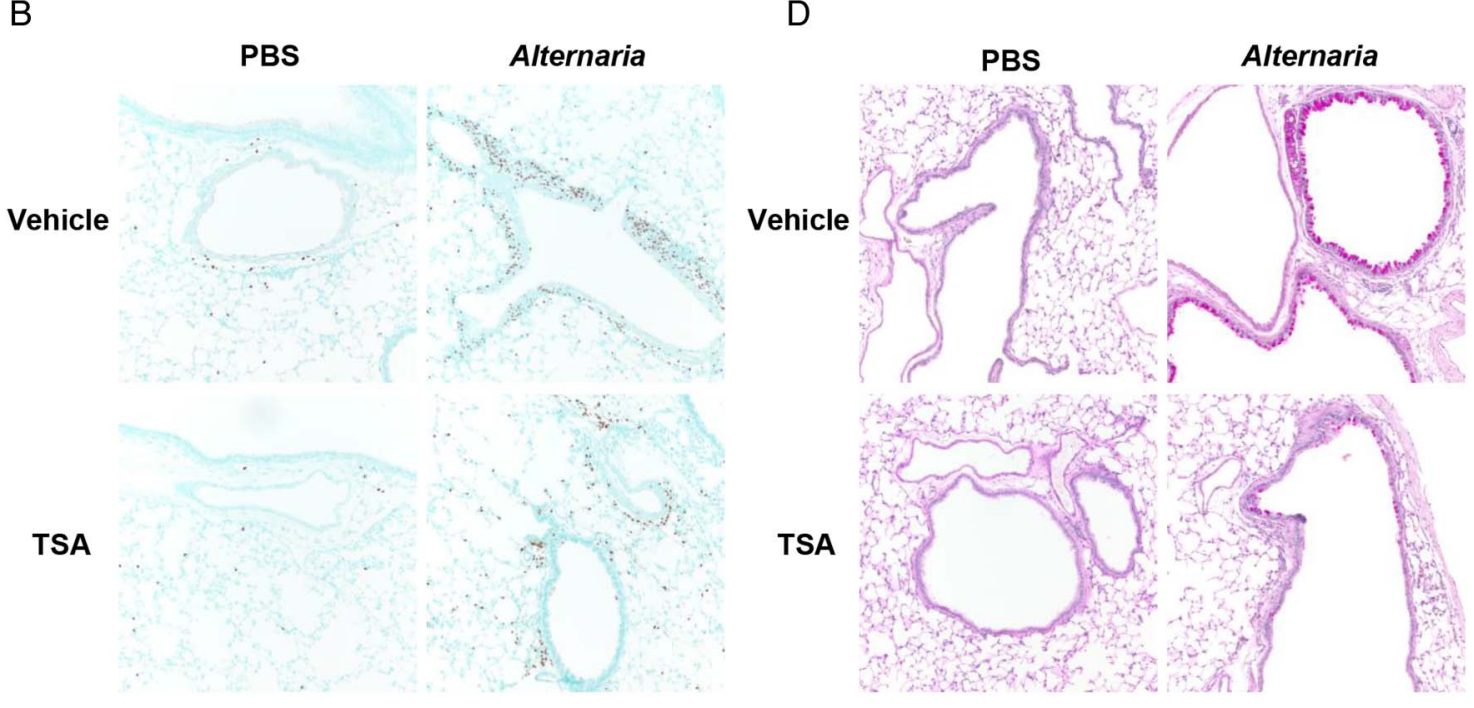

C
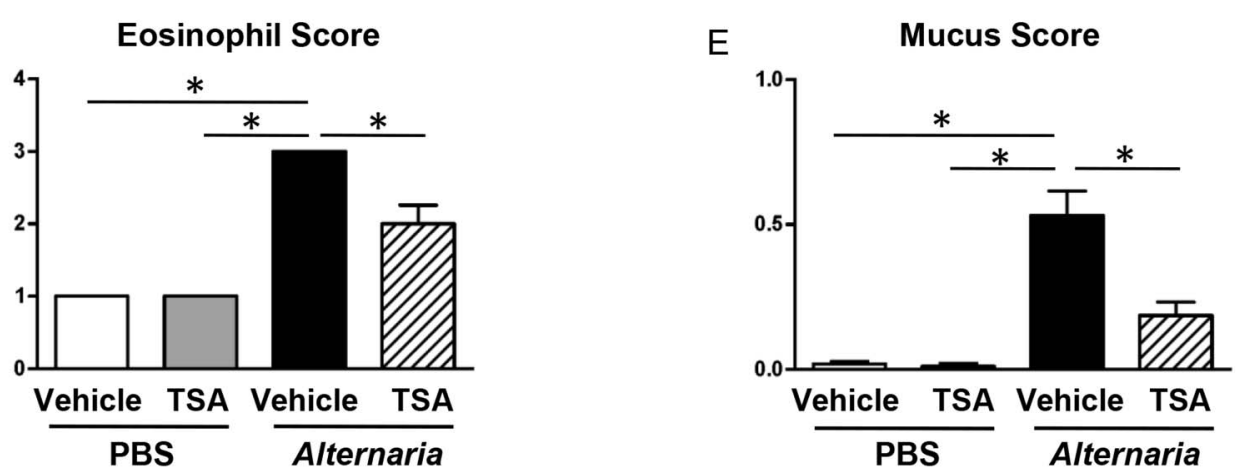

Figure 3 (A) Cell differentials from BAL fluids harvested $24 \mathrm{~h}$ after the last Alternaria extract challenge. (B) Representative sections and (C) eosinophil score as determined by anti-major basic protein (MBP) antibody staining $24 \mathrm{~h}$ after the last Alternaria extract challenge. (D) Representative sections and (E) mucus score as determined by periodic acid-Schiff's (PAS) staining $48 \mathrm{~h}$ after the last Alternaria extract challenge. The results are shown as mean \pm SEM of four mice in PBS-challenged groups and six mice in Alternaria extract-challenged groups. ${ }^{*} p<0.05$.

treatment suppressed the Alternaria extract-induced cytokine expressing ILC2 compared with vehicle treatment. Next, we measured IL-5 and IL-13 protein in BAL fluid and lung homogenates. Alternaria extract challenge significantly increased the protein expression of IL-5 $(\mathrm{p}<0.05)$ and IL-13 $(\mathrm{p}<0.05)$ in both BAL fluids and lung homogenates compared with PBS-challenged groups. We found a statistically significant decrease in Alternaria extract-induced protein expression of IL-5 $(\mathrm{p}<0.05)$ and IL-13 $(\mathrm{p}<0.05)$ in both the BAL fluids and lung homogenates from TSA-treated mice compared with vehicle-treated mice (figure 2). Further, there was a statistically significant decrease in Alternaria extract-induced protein expression of eosinophil-recruiting chemokines, CCL11 $(p<0.05)$ and CCL24 $(p<0.05)$, in both the BAL fluids and lung homogenates from TSA-treated mice compared with vehicle-treated mice. Meanwhile, there was no statistically significant difference in protein expression of Th2-recruiting chemokines, CCL17 (TARC) and CCL22 (MDC), between TSA-treated and vehicle-treated groups after Alternaria extract challenge (figure 2). Further, we measured proinflammatory cytokine and chemokine expression in BAL fluid and lung homogenates. Alternaria extract challenge significantly increased the protein expression of the neutrophil-recruiting chemokine KC (CXCL1) $(\mathrm{p}<0.05)$ in both the BAL fluids and lung 
Figure 4 (A) Mice were challenged with Alternaria extract intranasally for four consecutive days. Liraglutide or its vehicle was administered subcutaneously on day -2 and -1 , and then every $4 \mathrm{~h}$ before and after Alternaria extract challenge on day 0 3. The BAL fluids and lungs were harvested 1 and $24 \mathrm{~h}$, respectively, after last Alternaria extract challenge. The protein level of IL-33 (B and C) and IL- $1 \alpha$ (D and E) in the BAL fluids and lung homogenates was measured by ELISA. The results are shown as mean \pm SEM of four mice in PBS-challenged groups and six mice in Alternaria extract-challenged groups. ${ }^{*} \mathrm{p}<0.05$.
A

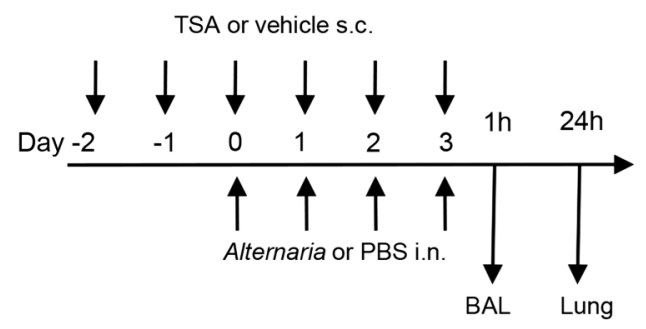

C

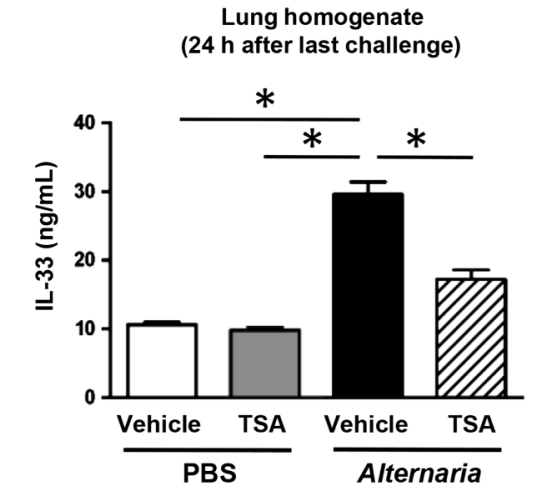

B

BAL fluid
(1 $\mathrm{h}$ after last challenge)

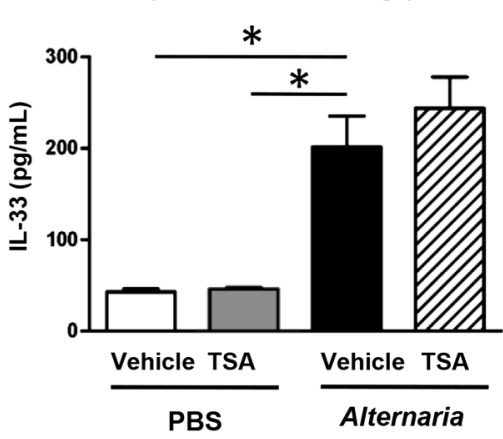

D

Lung homogenate (24 $\mathrm{h}$ after last challenge)

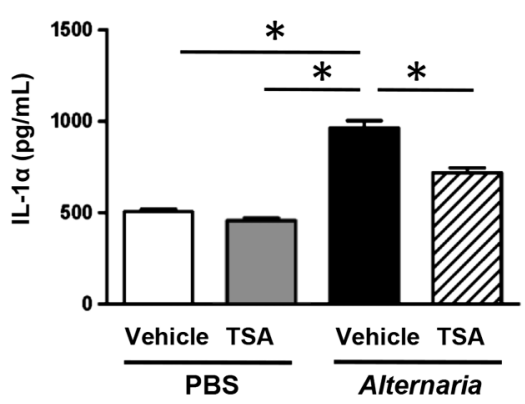

homogenates, TNF- $\alpha(\mathrm{p}<0.05)$ in the BAL fluids and granulocyte macrophage colony stimulating factor $(\mathrm{GM}-\mathrm{CSF})(\mathrm{p}<0.05)$ in the lung homogenates (see online supplementary figure S3). TSA treatment significantly decreased Alternaria extract-induced KC $(p<0.05)$ and GM-CSF $(p<0.05)$ in the lung homogenates, and TNF- $\alpha(\mathrm{p}<0.05)$ in the BAL fluids compared with vehicle treatment (see online supplementary figure S3). These results suggest that TSA treatment suppresses Alternaria extract-induced innate allergic immune and inflammatory responses.

\section{TSA treatment suppresses the number of perivascular eosinophils and mucus production in response to airway Alternaria extract challenge}

Based on our finding that TSA treatment decreased protein expression of IL-5, CCL11, and CCL24 in both BAL fluid and lung homogenates following Alternaria extract challenge for four consecutive days, we hypothesised that the reduction of IL-5, CCL11, and CCL24 by TSA treatment decreases eosinophil accumulation in the lung following Alternaria extract challenge. To test this hypothesis, we measured BAL cell counts and differentials in Alternaria extract-challenged or PBS-challenged mice treated with TSA or vehicle. Alternaria extract challenge significantly increased the number of total BAL cells, eosinophils, lymphocytes and neutrophils compared with PBSchallenged groups $(p<0.05)$. There was a statistically significant decrease in the number of total BAL cells, eosinophils, lymphocytes and neutrophils in the Alternaria extract-challenged mice treated with TSA compared with the Alternaria extractchallenged mice treated with vehicle $(\mathrm{p}<0.05)$ (figure $3 \mathrm{~A}$ ). However, there were no differences in the number of macrophages between the TSA-treated and vehicle-treated groups after Alternaria extract challenge. Next, we assessed perivascular eosinophils by immunohistopathology. Representative immunohistopathology sections stained with anti-MBP antibody are shown in figure 3B. We found that there were very few eosinophils around alveolar spaces and no perivascular eosinophils following intranasal PBS challenge, while there was a significant increase in perivascular eosinophils $(\mathrm{p}<0.05)$ in the mice challenged with Alternaria extract and challenged with vehicle. However, TSA treatment resulted in a significant decrease of perivascular eosinophils $(\mathrm{p}<0.05)$ in the lung challenged with Alternaria extract (figure 3C).

We also found that TSA treatment decreased lung protein expression of IL-13, a critical inducer of airway mucus production, ${ }^{25}$ in both BAL fluid and lung homogenates following Alternaria extract challenge (figure 2). Therefore, we tested the hypothesis that TSA treatment suppresses mucus production during innate immune responses to Alternaria extract. Representative histopathology sections stained with PAS solution are shown in figure 3D. There was no mucus or mucousproducing cells in the lungs of mice challenged with PBS. Alternaria extract challenge for four consecutive days significantly increased production and secretion of mucus $(p<0.05)$ from large airway epithelial cells $48 \mathrm{~h}$ after the last Alternaria extract challenge. However, TSA treatment significantly decreased the production and secretion of mucus $(p<0.05)$ compared with mice challenged with Alternaria extract treated with vehicle (figure 3E). Taken together, these results indicate that TSA treatment suppresses acute eosinophilic lung inflammation and airway mucus secretion in innate allergic responses to Alternaria extract challenge.

TSA treatment suppresses Alternaria extract-induced IL-33 in the lung homogenate, but not in BAL fluid

In this study, we found that TSA treatment suppressed the Alternaria extract-induced cytokine-expressing ILC2 compared with vehicle treatment. ILC2 are capable of producing large amounts of IL-5 and IL-13 following stimulation with 
IL-33, a combination of IL-33 and IL-25, TSLP, IL-2 or IL-7. ${ }^{5}$

The full-length IL-33 that has biological activity is released in the extracellular space after cell damage such as necrosis. ${ }^{26}$ Many airborne allergens contain proteases that induce rapid IL-33 release in BAL fluids after airway administration. ${ }^{726}$ First, we measured IL-33 protein level in BAL fluid and lung homogenates in the time course experiment after the last Alternaria extract challenge in the four consecutive days challenge protocol. The IL-33 protein level in BAL fluid peaked at $1 \mathrm{~h}$ after last Alternaria extract challenge; thereafter, the IL-33 protein level was decreased in a time-dependent manner (see online supplementary figure S4). In contrast, the IL-33 protein level in the lung homogenates was increased in a time-dependent manner and peaked at $24 \mathrm{~h}$. Therefore, we assayed IL-33 protein level in BAL fluid and lung homogenates 1 and $24 \mathrm{~h}$, respectively, after the last Alternaria extract challenge to determine the effect of TSA treatment (figure 4A). Alternaria extract challenge significantly increased the protein level of IL-33 $(p<0.05)$ in BAL fluid compared with PBS-challenged groups $1 \mathrm{~h}$ after the last challenge. However, the protein level of IL-33 in BAL fluid was not changed between TSA-treated and vehicle-treated groups with Alternaria extract challenge (figure 4B). On the other hand, we found a statistically significant decrease in Alternaria extract-induced protein expression of IL-33 $(\mathrm{p}<0.05)$ in lung homogenates from TSA-treated mice compared with vehicletreated mice (figure 4C). These results suggest that TSA treatment suppresses the expression of IL-33 in the lung but does not suppress the IL-33 release in the airway.

It has been reported that IL-33 expression is controlled by IL- $1 \alpha .{ }^{27}$ We therefore measured IL- $1 \alpha$ protein level in the lung homogenate $24 \mathrm{~h}$ after the last Alternaria extract challenge. Alternaria extract challenge significantly increased the protein level of IL-1 $\alpha(p<0.05)$ in the lung homogenates compared with PBS challenge $1 \mathrm{~h}$ after the last challenge. We found a statistically significant decrease in Alternaria extract-induced protein expression of IL-1 $\alpha \quad(p<0.05)$ in lung homogenates from TSA-treated mice compared with vehicle-treated mice (figure $4 \mathrm{D})$. This result suggests that intracellular IL- $1 \alpha$ expression may be associated with IL-33 expression in the lung.

\section{TSA treatment suppresses IL-33 expression in the lung epithelial cells}

We found that TSA treatment suppressed protein expression of IL-33 in lung homogenate $24 \mathrm{~h}$ after the last Alternaria extract challenge. To investigate the localisation of IL-33 expression and the effect of TSA on per cell IL-33 expression level, we used IL- $33^{\text {Citrine/+ }}$ reporter mice. There was expression of citrine in the lung from naïve IL-33 ${ }^{\text {Citrine/+ }}$ reporter mice, but no citrine expression was observed in the lung from naïve WT mice (see online supplementary figure S5A). We found that $\mathrm{CD}^{-}$EpCAM $^{+}$cells expressed citrine fluorescence in PBS-challenged mouse lungs by flow cytometry (figure 5A,B). There was a significant increase in the mean fluorescent intensity (MFI), percentage and number of the lung $\mathrm{CD}_{4} 5^{-} \mathrm{EpCAM}^{+}$ citrine $^{+}$cells in the Alternaria-challenged mice treated with vehicle compared with PBS-challenged mice treated with vehicle $(p<0.05)$. However, TSA treatment resulted in a decrease of the MFI, percentage and number in the lung CD45- $\mathrm{EpCAM}^{+}$ citrine $^{+}$cells compared with Alternaria extract-challenged mice that were treated with vehicle $(\mathrm{p}<0.05)$ (figure $5 \mathrm{~B}, \mathrm{C})$. In contrast, citrine fluorescence was not detected in $\mathrm{T}$ cells, B cells, DCs, macrophages, eosinophils or the other granulocytes (see online supplementary figure S5B). These results indicate that
TSA treatment reduced the number of lung epithelial cells expressing IL-33 following Alternaria extract challenge and decreased the amount of IL-33 expressed by individual cells in response to this same challenge.

\section{TSA treatment suppresses the number of mouse lung ILC2-producing IL-5 and IL-13 in response to airway rmIL-33 challenge}

In this study, there was no difference of IL-33 protein level in the BAL fluid between TSA-treated and vehicle-treated mice $1 \mathrm{~h}$ after last Alternaria extract challenge (figure 4B); meanwhile, TSA treatment suppressed the Alternaria extract-induced IL-5expressing and IL-13-expressing ILC2 compared with vehicle treatment (figure 1B). These data suggest that TSA treatment decreases ILC2 activation by inhibiting IL-33-induced activation of ILC2, rather than by inhibiting Alternaria extract-induced IL-33 release by epithelial cells. Therefore, we tested the hypothesis that TSA treatment suppresses IL-33-mediated ILC2 activation in vivo. To determine the effect of TSA on IL-33-mediated ILC2 activation, we enumerated the number of IL-5-expressing and IL-13-expressing lung ILC2 following either four consecutive days of rmIL-33 challenge or the vehicle for rmIL-33 in TSA-treated or vehicle-treated mice (figure 6A). rmIL-33 challenge significantly increased the number of lung ILC2 that expressed either IL-5 or IL-13 compared with vehicle-challenged mice $(\mathrm{p}<0.05)$ (figure $6 \mathrm{~B})$. There was a statistically significant decrease in the number of IL-5-expressing and IL-13-expressing ILC2 in the rmIL-33-challenged mice treated with TSA compared with the rmIL-33-challenged mice treated with the vehicle for TSA $(p<0.05)$ (figure $6 \mathrm{~B})$. Figure $6 \mathrm{C}$ shows the representative flow cytometry dot plots of IL-5 and IL-13 intracellular staining in the lung ILC2 from mice challenged with rmIL-33 or its vehicle following TSA treatment or TSA-vehicle treatment. This result indicates that TSA treatment inhibits IL-33-induced activation of ILC2.

\section{TSA treatment suppresses rmIL-33-induced allergic pulmonary inflammation}

We assessed cell differentials and cytokine and chemokine expression level in the BAL fluid and lung homogenates $24 \mathrm{~h}$ after rmIL-33 challenge for four consecutive days (figure 6A). IL-1 $\alpha$ protein expression was not changed by exogenous rmIL-33 challenge in lung homogenate (figure 7A). This result is consistent with a previous report that IL-33 neutralisation by soluble ST2 administration had no effect in house dust mite extract-induced lung IL-1 $\alpha$ protein expression in mouse model. ${ }^{27}$ On the other hand, protein expression of IL-5 and IL-13 in both BAL fluids and lung homogenates was significantly increased in rmIL-33-challenged mice compared with vehiclechallenged mice $(\mathrm{p}<0.05)$; and TSA treatment significantly decreased the rmIL-33-induced IL-5 and IL-13 protein expression compared with TSA-vehicle treatment $(\mathrm{p}<0.05)$ (figure 7B). CCL11 and CCL24 were also increased in lung homogenates from rmIL-33-challenged mice compared with its vehiclechallenged mice $(\mathrm{p}<0.05)$; and TSA treatment significantly decreased the rmIL-33-induced CCL11 and CCL24 expression in the lung homogenates compared with treatment with the vehicle for TSA $(p<0.05)$ (figure 7B). rmIL-33 challenge significantly induced CCL17 and CCL22 expression in the lung homogenates $(p<0.05)$, but TSA treatment did not decrease these chemokines compared with treatment with the vehicle for TSA (figure 7B). Further, there was a statistically significant decrease in the number of total BAL cells and eosinophils in the rmIL-33-challenged mice treated with TSA compared with the 
Figure 5 (A) Representative histograms of citrine (IL-33) expression on $\mathrm{CD}^{4} 5^{-} \mathrm{EpCAM}^{+}$cells in the lungs from WT mice (filled grey area) or IL-33 ${ }^{\text {Citrine/+ }}$ reporter mice challenged with vehicle-PBS (solid black line), trichostatin A (TSA)-PBS (dotted green line), vehicle-Alternaria extract (solid blue line) and TSA-Alternaria extract (dotted orange line). (C) The MFI, the percentage and the number of Citrine ${ }^{+}$ $\mathrm{CD}^{-} 5^{-} \mathrm{EpCAM}^{+}$cells in the lung of mice challenged with PBS or Alternaria extract following vehicle or TSA treatment. The results are shown as mean \pm SEM of six mice in

PBS-challenged groups and seven mice in Alternaria extract-challenged groups. ${ }^{*} \mathrm{p}<0.05$.
A

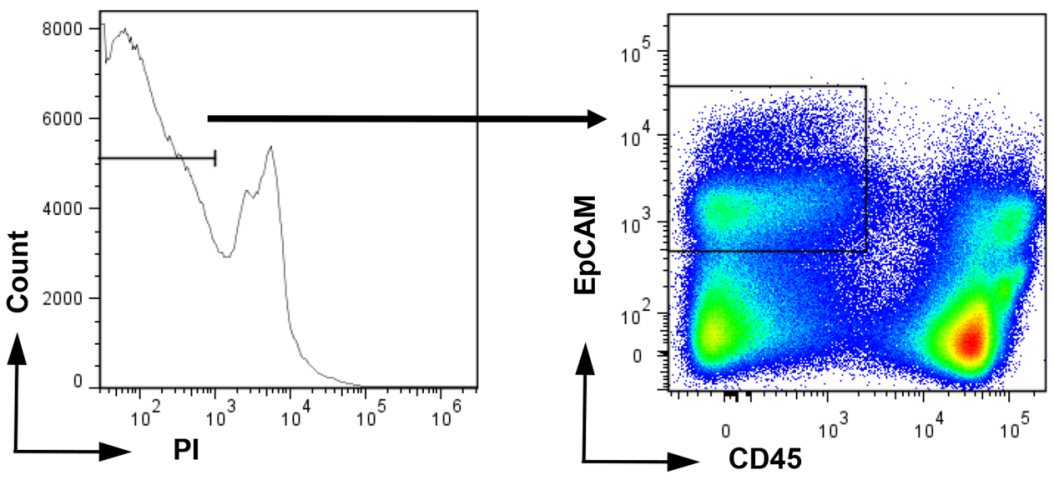

B

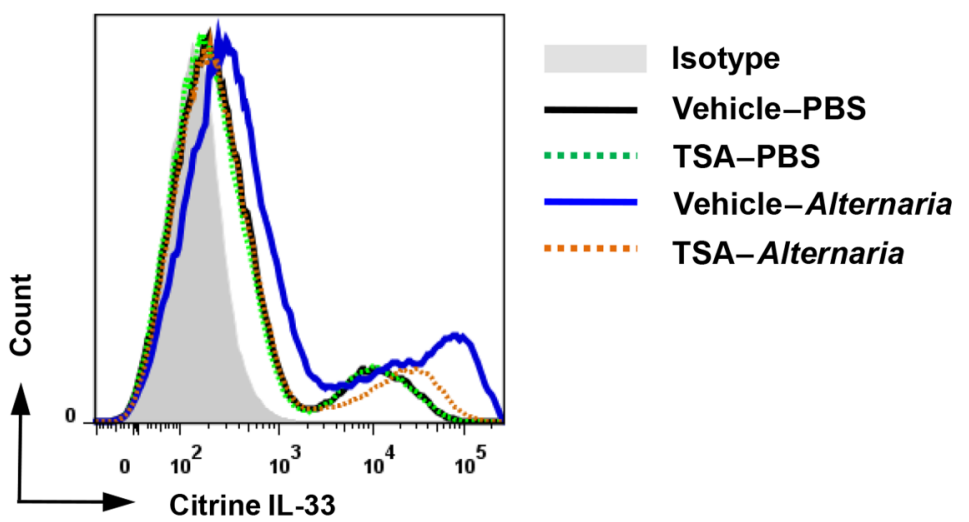

C

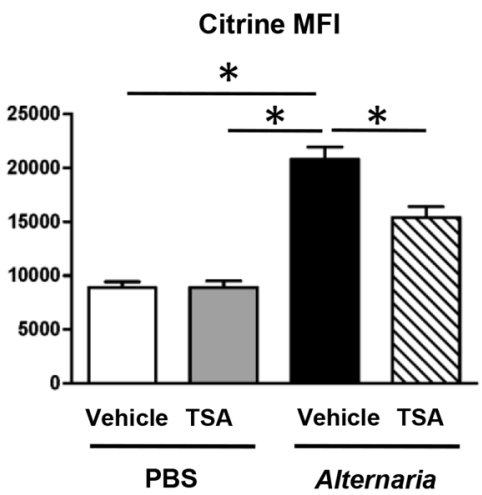

$\%$ of Citrine ${ }^{+}$cells / total EC

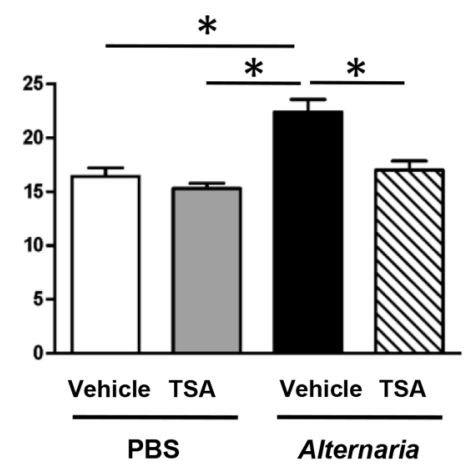

Number of Citrine ${ }^{+}$cells

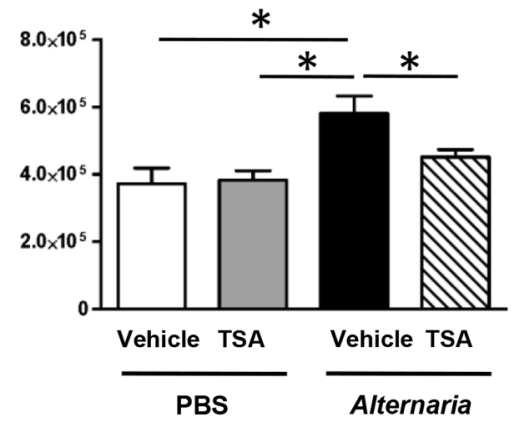

rmIL-33-challenged mice treated with vehicle $(p<0.05)$ (figure $7 \mathrm{C}$ ). These results suggest that TSA treatment suppresses IL-33-induced Th2 cytokine and eosinophil chemotactic chemokine expression, and subsequent eosinophil accumulation in the airway.
TSA treatment concomitant with Alternaria extract challenge suppresses allergic pulmonary inflammation

To determine the effect of TSA treatment as a potential therapeutic procedure, we administered TSA or its vehicle concomitantly with Alternaria extract challenge (figure 8A). We found a 
A TSA or vehicle s.c.

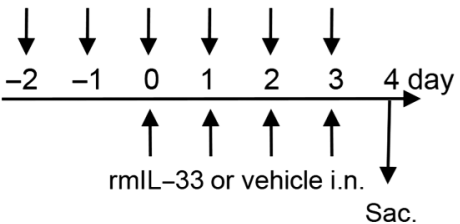

B
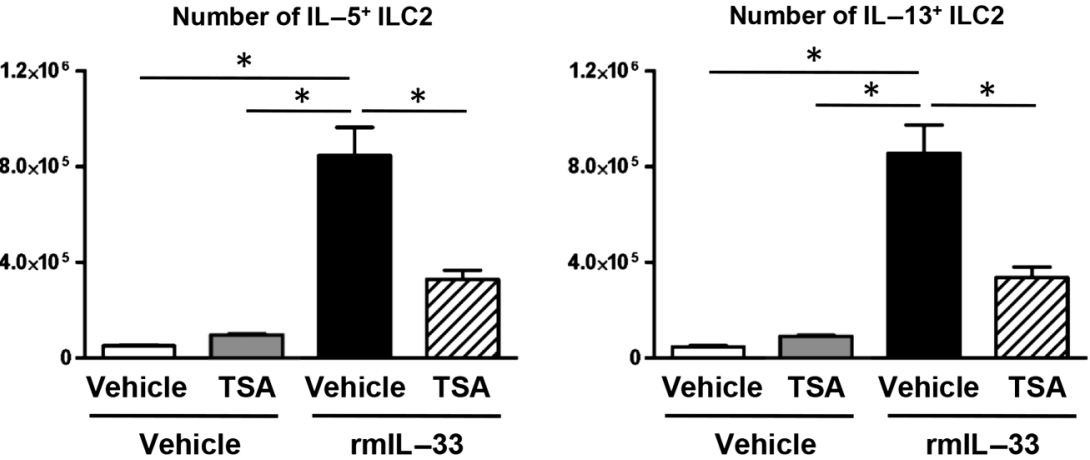

C
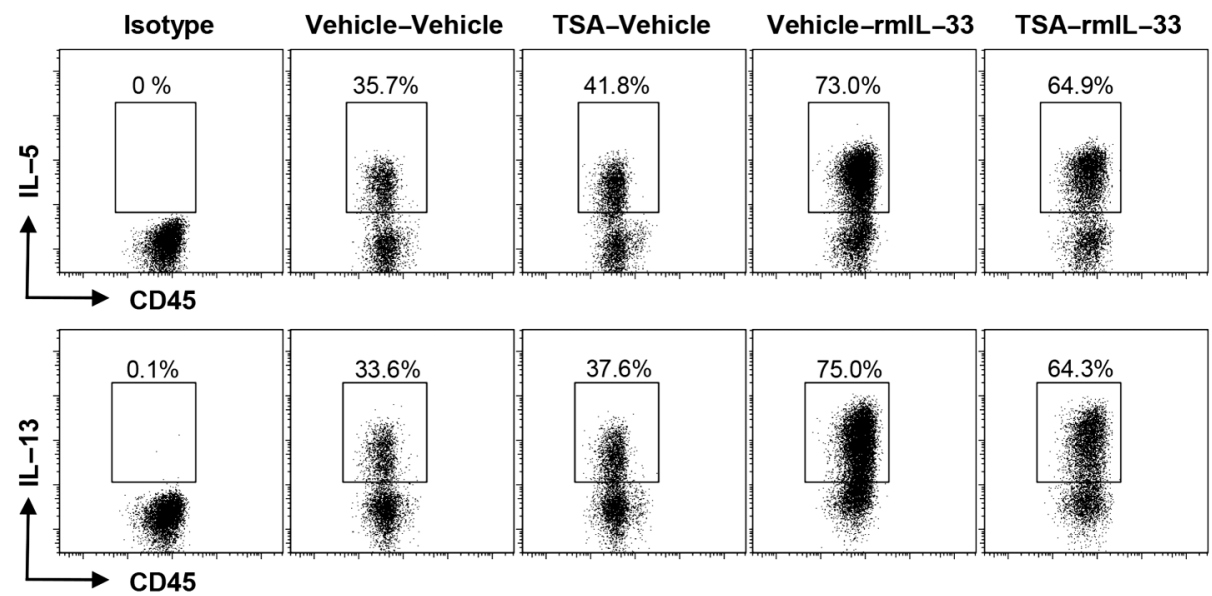

Figure 6 (A) Mice were challenged with rmIL-33 or its vehicle intranasally for four consecutive days. Trichostatin A (TSA) or its vehicle was administered subcutaneously on day -2 and -1 , and then every $1 \mathrm{~h}$ before rmIL-33 challenge on day $0-3$. (B) The number of IL-5-expressing and IL-13-expressing ILC2 in the lung from the mice challenged with rmIL-33 or its vehicle following TSA or TSA-vehicle treatment. (C) Representative plots of IL-5 and IL-13 intracellular staining in ILC2 in the lungs of mice challenged with rmIL-33 or its vehicle following TSA or TSA-vehicle treatment. The results are shown as mean \pm SEM of four mice in PBS-challenged groups and six mice in Alternaria extract-challenged groups. ${ }^{*} p<0.05$.

statistically significant decrease in Alternaria extract-induced protein expression of IL-33 $(\mathrm{p}<0.05)$ in lung homogenates from TSA-treated mice compared with vehicle-treated mice, but there was no change in Alternaria extract-induced IL- $1 \alpha$ expression between TSA-treated mice and vehicle-treated mice (figure 8B). Further, we found a statistically significant decrease in Alternaria extract-induced protein expression of IL-5, IL-13, CCL11 and CCL24 in both the BAL fluids and lung homogenates from TSA-treated mice compared with vehicle-treated mice $(\mathrm{p}<0.05)$ (figure $8 \mathrm{C})$. Meanwhile, there was no statistically significant difference in protein expression of CCL17 and CCL22, between TSA-treated and vehicle-treated groups with Alternaria extract challenge (figure $8 \mathrm{C}$ ). In addition, there was a statistically significant decrease in the number of total BAL cells and eosinophils in the Alternaria extract-challenged mice treated with TSA compared with the Alternaria extract-challenged mice treated with vehicle $(\mathrm{p}<0.05)$ (figure $8 \mathrm{D}$ ). These results suggest that concomitant TSA treatment also suppresses Alternaria extract-induced innate eosinophilic airway inflammation.

\section{DISCUSSION}

In this study, we report for the first time that an HDACi significantly decreases the early innate immune responses to aeroallergen challenge mediated by ILC2 activation. TSA treatment significantly decreased the number of lung ILC2-expressing IL-5 and IL-13 after four consecutive days Alternaria extract challenge. In addition, TSA decreased IL-5, IL-13, CCL11 and CCL24 protein expression, the number of eosinophils, lymphocytes and neutrophils in the BAL fluid, and perivascular eosinophil accumulation induced by Alternaria extract challenge. Further, mucus score in the airways $48 \mathrm{~h}$ after the last Alternaria 

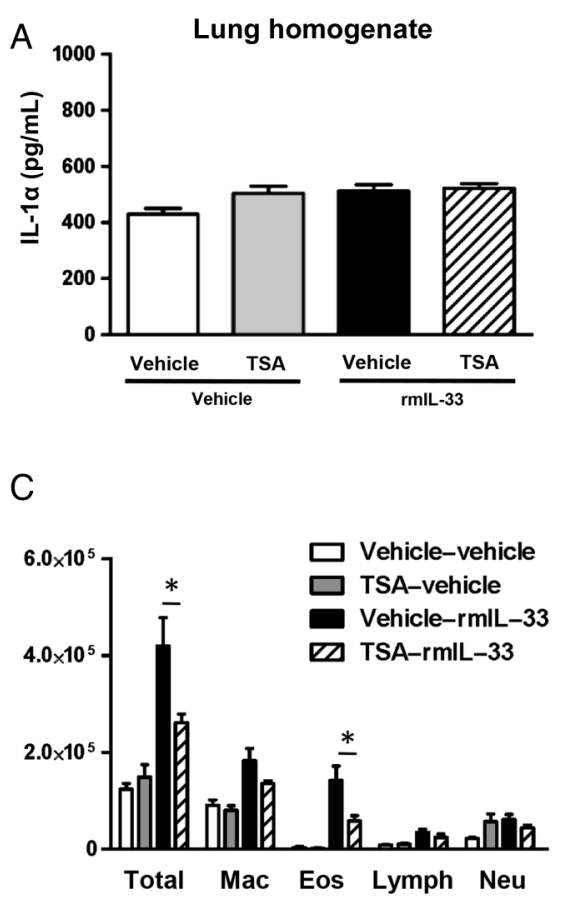
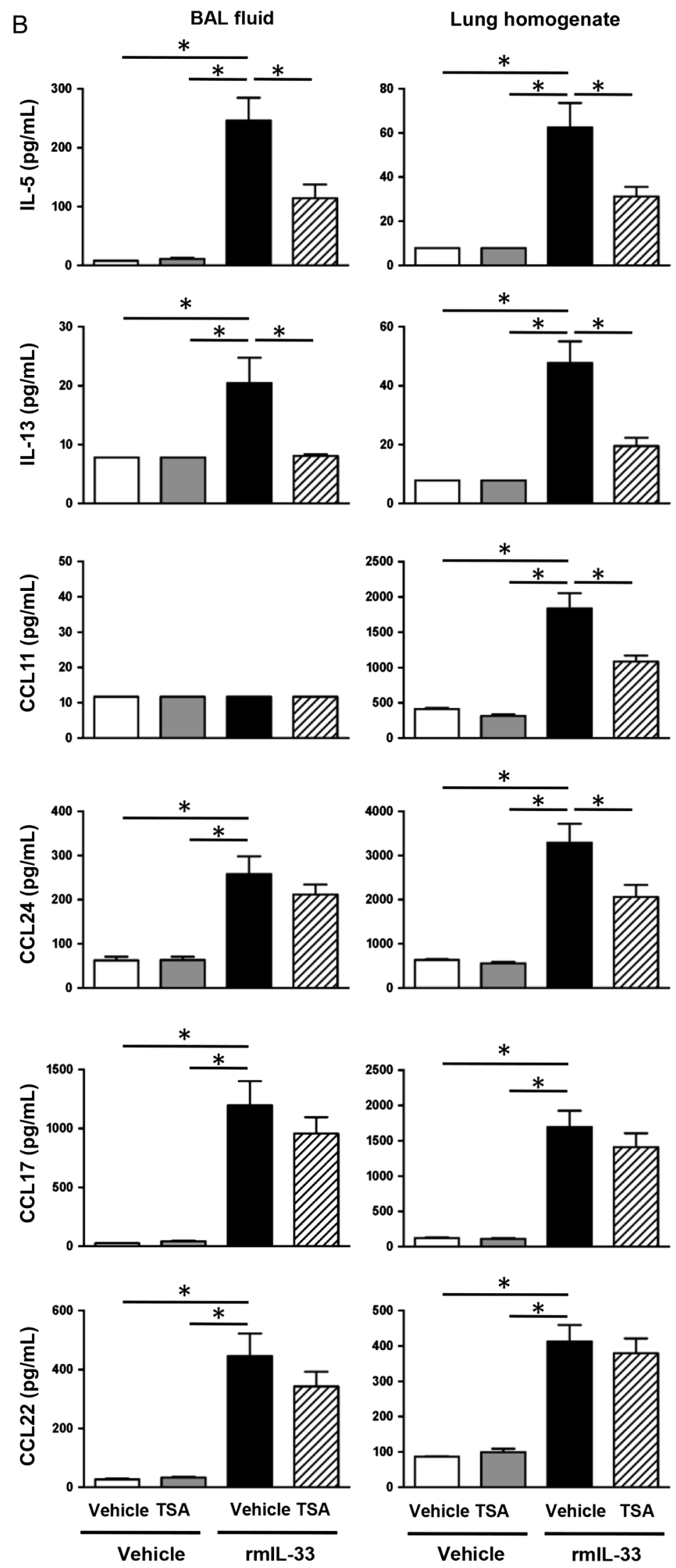

Figure 7 (A and B) The BAL fluids and lungs were harvested $24 \mathrm{~h}$ after last rmIL-33 challenge to measure the protein expression of IL-1 $\alpha, \mathrm{IL}-5$, IL-13, CCL11, CCL17, CCL22 and CCL24. (C) Cell differentials from BAL fluids harvested $24 \mathrm{~h}$ after the last rmlL-33 challenge. The results are shown as mean \pm SEM of four mice in PBS-challenged groups and 11 mice in rmIL-33-challenged groups. * $p<0.05$. 
A

TSA or vehicle s.c.

and

Alternaria or PBS i.n.

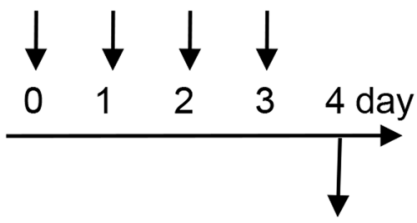

Sac.

B
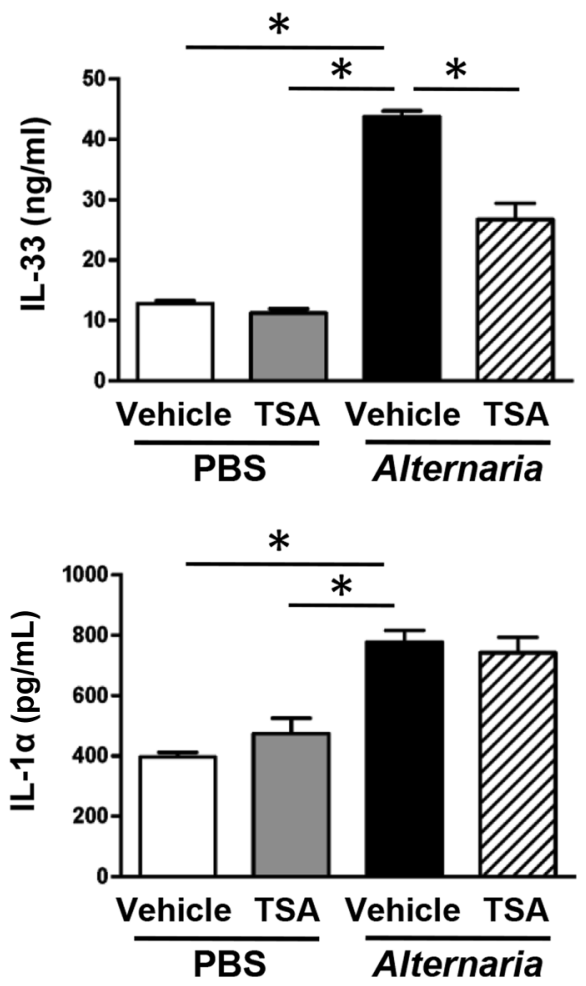

D

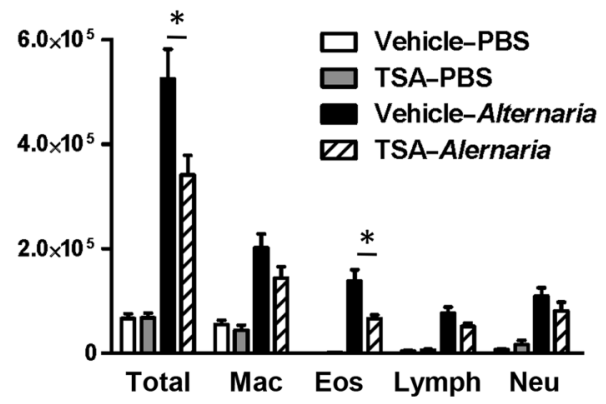

C
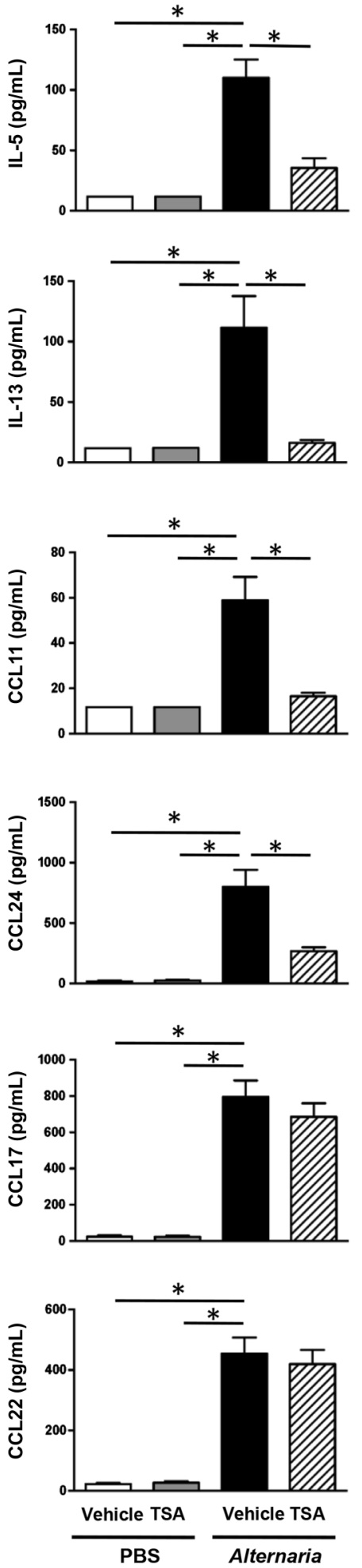
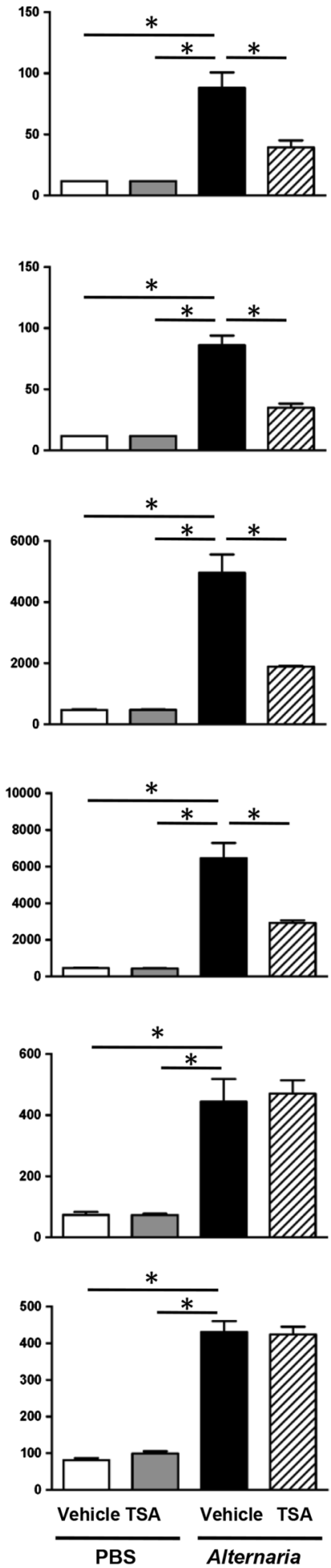

Figure 8 (A) Mice were challenged with Alternaria extract and trichostatin A (TSA) concomitantly for four consecutive days. (B and C) The BAL fluids and lungs were harvested $24 \mathrm{~h}$ after last Alternaria extract challenge to measure the protein expression of IL-1 $\alpha, \mathrm{IL}-5, \mathrm{IL}-13$, IL-33, CCL11, CCL17, CCL22 and CCL24. (D) Cell differentials from BAL fluids harvested $24 \mathrm{~h}$ after the last Alternaria extract challenge. The results are shown as mean \pm SEM of four mice in PBS-challenged groups and six mice in Alternaria extract-challenged groups. ${ }^{*} p<0.05$.

extract challenge was downregulated by TSA treatment. Although TSA treatment did not decrease Alternaria extract-induced IL-33 release in the BAL fluids $1 \mathrm{~h}$ after the last Alternaria extract challenge, Alternaria extract-induced IL-33 and IL-1 $\alpha$ protein level in the lung homogenates $24 \mathrm{~h}$ after the last Alternaria extract challenge was significantly decreased by TSA treatment. IL-33 expression was localised in lung epithelial cells. Furthermore, TSA treatment significantly decreased the number of lung ILC2-expressing IL-5 and IL-13, the protein expression of IL-5, IL-13, CCL11 and CCL24 in the lung 
homogenates and the number of eosinophils in the BAL fluids after four consecutive days of exogenous rmIL-33 challenge.

Epigenetic regulation plays an important role in immune responses. ${ }^{28}$ However, the mechanisms by which HDAC inhibition regulates immune responses are still unclear.

Since histone acetylation regulates DNA-templated processes by influencing local chromatin structure, ${ }^{11}$ transcriptional activation is generally correlated with histone acetylation and repression is associated with histone deacetylation. ${ }^{29}{ }^{30}$ However, histone acetylation levels differ in cell types or regions of tissue. ${ }^{31}$ Since previous studies have shown that effects of HDACi were dependent on the type of diseases and the type of HDAC inhibition, the therapeutic effects of HDACi treatment for inflammatory or allergic diseases remain controversial. Several studies reported that TSA enhances inflammatory gene expression mediated by NF- $\mathrm{KB}^{14-16}$; meanwhile, we found that TSA treatment decreased Alternaria extract-induced proinflammatory cytokines and chemokines such as KC, TNF- $\alpha$ and GM-CSF (see online supplementary figure S3), as well as type 2 cytokine and chemokine such as IL-5, IL-13 and eotaxin (figure 2). Further, many studies have reported that HDACi have strong anti-inflammatory effects as well as antitumor effects. For instance, TSA attenuated OVA sensitisation and challengeinduced allergic airway inflammation by reducing IgE levels, mucus and proinflammatory cell infiltration scores, and airway hyperresponsiveness in mouse models of adaptive immunitymediated OVA-induced inflammation. ${ }^{20}{ }^{21}$ In addition, HDACi treatment ameliorated collagen-induced rheumatoid arthritis ${ }^{32}$ and DNFB-induced allergic contact dermatitis ${ }^{33}$ in a rodent model. Our finding and these animal studies suggest the possibility of novel therapeutic strategies for chronic allergic diseases using HDACi treatment.

Previous toxicity and dose-ranging studies in vivo in rats showed that TSA was well tolerated in single doses of up to $5 \mathrm{mg} / \mathrm{kg}$ administered by subcutaneous injection. ${ }^{34}$ In our mouse model of four consecutive days of Alternaria extract challenge, an anti-inflammatory effect without toxicity was observed at doses of both 0.5 and $2.0 \mathrm{mg} / \mathrm{kg}$ TSA; however, there was no statistically significant difference in protein expression of IL-5 and IL-13 between $0.5 \mathrm{mg} / \mathrm{kg}$ TSA-treated and vehicle-treated groups after Alternaria extract challenge (data not shown). $2.0 \mathrm{mg} / \mathrm{kg}$ TSA treatment significantly decreased the lung protein expression of IL-5 and IL-13 compared with vehicle treatment (figure 2). Therefore, we investigated the effect of TSA at the dose of $2.0 \mathrm{mg} / \mathrm{kg}$ in subsequent experiments.

In this study, we focused on ILC2, a cell type that produces high levels of Th2 cytokines such as IL-5 and IL-13 in response to IL-33. IL-33 is a nuclear protein constitutively expressed in epithelial cells, and is released as an endogenous alarm signal, known as an alarmin, in response to tissue damage. ${ }^{26} 35$ This nuclear cytokine has been reported as a pathogenic factor in several inflammatory diseases such as asthma, arthritis, inflammatory bowel disease and multiple sclerosis. ${ }^{36}{ }^{37}$ Recent studies revealed that IL-33 is a critical cytokine to expand ILC2 proliferation and activation. ${ }^{45}$ We found that IL-33 protein release in the BAL fluids $1 \mathrm{~h}$ after 4 th Alternaria extract challenge was not different between TSA and its vehicle treatment. On the other hand, TSA treatment significantly decreased the number of ILC2-expressing IL-5 and IL-13 $24 \mathrm{~h}$ after four consecutive days of Alternaria extract challenge or rmIL-33 challenge. These results suggest that TSA inhibits IL-33-induced ILC2 activation but does not attenuate early IL-33 release by Alternaria extract challenge. These are critical findings because it reveals a suppressive effect by HDACi on IL-33-induced ILC2 activation and its subsequent attenuation of innate allergic immune responses.

We further found that IL-1 $\alpha$ expression in the lung homogenates was increased by Alternaria extract challenge, but not exogenous rmIL-33 challenge, and TSA significantly decreased the Alternaria extract-induced IL-1 $\alpha$ expression (figures 4D and $7 \mathrm{~A})$. IL-1 $\alpha$ is also known as an endogenous alarmin to induce inflammation and initiates an autocrine feedback for the expression of other cytokines, including GM-CSF and IL-33. ${ }^{27}$ We found that TSA suppressed lung IL-33 expression in epithelial cells after four consecutive days Alternaria extract challenge (figure 4C). Taken together, IL-1 $\alpha$ expression level may be associated with intracellular IL-33 expression in lung epithelial cells.

Currently, there are no HDACi approved for the treatment of allergic diseases. However, HDACi have been investigated in rodent models of allergic inflammation. A recent study reported that a selective HDAC6 inhibitor (tubastatin) showed antiinflammatory and antirheumatic effects in rodent models. ${ }^{38}$ Further, the oral administration of another selective HDAC6 inhibitor compound, ACY-1215, attenuated TNCB-induced skin inflammation, including contact hypersensitivity. ${ }^{39}$ In vitro studies suggest possible mechanisms by which HDACi suppress the activity of inflammatory cells or epithelial cells. HDAC6, a member of the cytoplasmic class IIb HDACs, is a unique enzyme that deacetylates non-histone proteins, such as $\alpha$-tubulin and heat shock protein 90 (Hsp90). HDAC6 is a key protein involved in aggresome formation that may act as an adaptor protein linking polyubiquitinated proteins to the dynein motor complex for transport. Inhibition of HDAC6 blocks aggresome formation. ${ }^{40}$ In addition, inhibition of HDAC6 results in hyperacetylation of Hsp90; consequently, inactivation of Hsp90 chaperone activity leads facilitating the structural maturation and complex assembly of client proteins including steroid hormone receptors and selected kinases. ${ }^{41}$ Since HDAC6 enzymatic activity seems to be required for the regulation of cell morphology, HDAC6 activity in lymphocytes may regulate cell migration and immune synapse formation. ${ }^{4}$

In this study, we showed that HDAC inhibition by TSA reduces innate allergic airway inflammation by inhibiting ILC2 activation using a mouse model of four consecutive days of Alternaria extract challenge or rmIL-33 challenge. Further, the inhibition of ILC2 activation attenuated eosinophilia in the lung and mucus hypersecretion from the airway epithelial cells. These results suggest that histone acetylation is involved in the control of development of allergic airway inflammation mediated by ILC2 activation stimulated with IL-33. HDACi drugs require further experimental analysis before their use in human clinical trials, but our results suggest a promising therapeutic effect for the treatment of allergic lung disorders.

Acknowledgements The authors are grateful to Dr Andrew N. J. McKenzie, MRC Laboratory of Molecular Biology, Cambridge University, Cambridge, UK, for providing IL-33 ${ }^{\text {Citrine/Citrine }}$ mice.

Contributors ST designed and performed experiments, analysed data and wrote the manuscript. KG and SR contributed to the acquisition of data. WZ, DCN, MHB and MTS advised and contributed to the overall development of this study and helped finalise the manuscript. KLB and VVP contributed to the analysis interpretation of histopathology data. SS and RSP designed and supervised the research and edited the manuscript.

Funding This work was supported by Department of Veterans Affairs 2101BX000624, NIH Al 095227-02, Al 111820, HL 090664-04, HL122554 and Al 121420.

Competing interests None declared.

Provenance and peer review Not commissioned; externally peer reviewed. 
Open Access This is an Open Access article distributed in accordance with the Creative Commons Attribution Non Commercial (CC BY-NC 4.0) license, which permits others to distribute, remix, adapt, build upon this work non-commercially, and license their derivative works on different terms, provided the original work is properly cited and the use is non-commercial. See: http://creativecommons.org/ licenses/by-nc/4.0/

\section{REFERENCES}

1 Postma DS, Timens W. Remodeling in asthma and chronic obstructive pulmonary disease. Proc Am Thorac Soc 2006;3:434-9.

2 Barnes PJ. Immunology of asthma and chronic obstructive pulmonary disease. Nat Rev Immunol 2008:8:183-92.

3 Wenzel SE. Asthma phenotypes: the evolution from clinical to molecular approaches. Nat Med 2012;18:716-25.

4 Neill DR, Wong SH, Bellosi A, et al. Nuocytes represent a new innate effector leukocyte that mediates type-2 immunity. Nature 2010;464:1367-70.

5 Halim TYF, Krauß RH, Sun AC, et al. Lung Natural Helper Cells Are a Critical Source of Th2 Cell-Type Cytokines in Protease Allergen-Induced Airway Inflammation. Immunity 2012;36:451-63.

6 Barlow JL, McKenzie ANJ. Type-2 innate lymphoid cells in human allergic disease. Curr Opin Allergy Clin Immunol 2014;14:397-403.

7 Snelgrove RJ, Gregory LG, Peiró T, et al. Alternaria-derived serine protease activity drives IL-33-mediated asthma exacerbations. J Allergy Clin Immunol 2014;134:583-92.e6.

8 Kabata H, Moro K, Fukunaga K, et al. Thymic stromal lymphopoietin induces corticosteroid resistance in natural helper cells during airway inflammation. Nat Commun 2013:4:2675.

9 Adcock IM, Tsaprouni L, Bhavsar P, et al. Epigenetic regulation of airway inflammation. Curr Opin Immunol 2007;19:694-700.

10 Dekker FJ, van den Bosch T, Martin NI. Small molecule inhibitors of histone acetyltransferases and deacetylases are potential drugs for inflammatory diseases. Drug Discov Today 2014;19:654-60.

11 Kazantsev AG, Thompson LM. Therapeutic application of histone deacetylase inhibitors for central nervous system disorders. Nat Rev Drug Discov 2008;7:854-68.

$12 \mathrm{Kim}$ HJ, Bae SC. Histone deacetylase inhibitors: molecular mechanisms of action and clinical trials as anti-cancer drugs. Am J Trans/ Res 2011:3:166-79.

13 Mottamal M, Zheng S, Huang TL, et al. Histone deacetylase inhibitors in clinical studies as templates for new anticancer agents. Molecules 2015:20:3898-941.

14 Chen Lf, Fischle W, Verdin E, et al. Duration of nuclear NF-kappa B action regulated by reversible acetylation. Science 2001;293:1653-7.

15 Adam E, Quivy V, Bex F, et al. Potentiation of tumor necrosis factor-induced NF- $\kappa B$ activation by deacetylase inhibitors is associated with a delayed cytoplasmic reappearance of $\mid \kappa B \alpha$. Mol Cell Biol 2003;23:6200-9.

16 Gilmour PS, Rahman I, Donaldson K, et al. Histone acetylation regulates epithelial IL-8 release mediated by oxidative stress from environmental particles. Am J Physiol Lung Cell Mol Physiol 2003;284:L533-40.

17 Han SB, Lee JK. Anti-inflammatory effect of Trichostatin-A on murine bone marrow-derived macrophages. Arch Pharm Res 2009;32:613-24.

18 Halili MA, Andrews MR, Labzin LI, et al. Differential effects of selective HDAC inhibitors on macrophage inflammatory responses to the Toll-like receptor 4 agonist LPS. J Leukoc Biol 2010;87:1103-14.

19 Suh HS, Choi S, Khattar P, et al. Histone deacetylase inhibitors suppress the expression of inflammatory and innate immune response genes in human microglia and astrocytes. J Neuroimmune Pharmacol 2010;5:521-32.

20 Choi JH, Oh SW, Kang MS, et al. Trichostatin A attenuates airway inflammation in mouse asthma model. Clin Exp Allergy 2005;35:89-96.
21 Royce SG, Dang W, Yuan G, et al. Effects of the histone deacetylase inhibitor, Trichostatin $A$, in a chronic allergic airways disease model in mice. Arch Immunol Ther Exp (Warsz) 2012;60:295-306.

22 Banerjee A, Trivedi CM, Damera G, et al. Trichostatin A abrogates airway constriction, but not inflammation, in murine and human asthma models. Am J Respir Cell Mol Biol 2012;46:132-8.

23 Hardman CS, Panova V, McKenzie ANJ. IL-33 citrine reporter mice reveal the temporal and spatial expression of IL-33 during allergic lung inflammation. Eur J Immunol 2013;43:488-98.

24 Denzler KL, Farmer SC, Crosby JR, et al. Eosinophil major basic protein-1 does not contribute to allergen-induced airway pathologies in mouse models of asthma. J Immunol 2000;165:5509-17.

25 Zhu Z, Homer RJ, Wang Z, et al. Pulmonary expression of interleukin-13 causes inflammation, mucus hypersecretion, subepithelial fibrosis, physiologic abnormalities, and eotaxin production. J Clin Invest 1999;103:779-88.

26 Cayrol C, Girard JP. IL-33: an alarmin cytokine with crucial roles in innate immunity, inflammation and allergy. Curr Opin Immunol 2014;31:31-7.

27 Willart MA, Deswarte K, Pouliot P, et al. Interleukin-1 $\alpha$ controls allergic sensitization to inhaled house dust mite via the epithelial release of GM-CSF and IL-33. J Exp Med 2012;209:1505-17

28 Licciardi PV, Karagiannis TC. Regulation of Immune Responses by Histone Deacetylase Inhibitors. ISRN Hematology 2012;2012:1-10.

29 Struhl K. Histone acetylation and transcriptional regulatory mechanisms. Genes Dev 1998;12:599-606.

30 Deckert J, Struhl K. Histone acetylation at promoters is differentially affected by specific activators and repressors. Mol Cell Biol 2001;21:2726-35.

31 Iwabata H, Yoshida M, Komatsu Y. Proteomic analysis of organ-specific post-translational lysine-acetylation and -methylation in mice by use of anti-acetyl lysine and -methyl lysine mouse monoclonal antibodies. Proteomics 2005:5:4653-64.

32 Lin $\mathrm{HS}, \mathrm{Hu} \mathrm{CY}, \mathrm{Chan} \mathrm{HY}$, et al. Anti-rheumatic activities of histone deacetylase (HDAC) inhibitors in vivo in collagen-induced arthritis in rodents. $\mathrm{Br} J$ Pharmacol 2007:150:862-72.

33 Shi YL, Gu J, Park JJ, et al. Histone deacetylases inhibitor Trichostatin A ameliorates DNFB-induced allergic contact dermatitis and reduces epidermal Langerhans cells in mice. J Dermatol Sci 2012;68:99-107.

34 Vigushin DM, Ali S, Pace PE, et al. Trichostatin A is a histone deacetylase inhibitor with potent antitumor activity against breast cancer in vivo. Clin Cancer Res 2001;7:971-6.

35 Palmer G, Gabay C. Interleukin-33 biology with potential insights into human diseases. Nat Rev Rheumatol 2011;7:321-9.

36 Pastorelli L, De Salvo C, Cominelli MA, et al. Novel cytokine signaling pathways in inflammatory bowel disease: insight into the dichotomous functions of IL-33 during chronic intestinal inflammation. Therap Adv Gastroenterol 2011;4:311-23.

37 Duan L, Chen J, Gong F, et al. The role of IL-33 in rheumatic diseases. Clin Dev Immunol 2013;2013:1-5.

38 Vishwakarma $\mathrm{S}$, Iyer LR, Muley $\mathrm{M}$, et al. Tubastatin, a selective histone deacetylase 6 inhibitor shows anti-inflammatory and anti-rheumatic effects. Int Immunopharmacol 2013:16:72-8.

39 Tsuji G, Okiyama N, Villarroel VA, et al. Histone deacetylase 6 inhibition impairs effector CD8 T-cell functions during skin inflammation. J Allergy Clin Immunol 2015;135:1228-39.

40 Olzmann JA, Li L, Chin LS. Aggresome formation and neurodegenerative diseases: therapeutic implications. Curr Med Chem 2008:15:47-60.

41 Kovacs JJ, Murphy PJM, Gaillard S, et al. HDAC6 regulates Hsp90 acetylation and chaperone-dependent activation of glucocorticoid receptor. Mol Cell 2005;18:601-7.

42 Valenzuela-Fernández A, Cabrero JR, Serrador JM, et al. HDAC6: a key regulator of cytoskeleton, cell migration and cell-cell interactions. Trends Cell Biol 2008;18:291-7. 